\title{
Phase-Sensitive Wavelength Conversion Based on Cascaded Quadratic Processes in Periodically Poled Lithium Niobate Waveguides
}

\author{
Sheng Liu, Kwang Jo Lee, Joseph Kakande, Francesca Parmigiani, Radan Slavik, Periklis Petropoulos, and \\ David J. Richardson \\ Optoelectronics Research Centre, University of Southampton, Southampton, SO17 1BJ, United Kingdom
}

Katia Gallo

Department of Applied Physics, Royal Institute of Technology (KTH), 10691 Stockholm, Sweden

shl@orc.soton.ac.uk

\begin{abstract}
We propose and experimentally demonstrate a novel scheme of phase-sensitive wavelength conversion, based on a combination of cascaded second-order nonlinear effects in two cascaded periodically poled lithium niobate waveguides.

(C2010 Optical Society of America

OCIS codes: (060.4510) Optical communications; (130.3730) Lithium niobate; (190.4410) Nonlinear optics, parametric processes; (230.4320) Nonlinear optical devices.
\end{abstract}

\section{Introduction}

Phase-sensitive amplifiers (PSAs) have the potential to play a critical role in the regeneration of phase encoded signals as they can suppress the cumulative linear and nonlinear phase noise added respectively by optical amplifiers and nonlinear effects in the fiber. Several PSA configurations have been demonstrated based on a number of technologies, such as four-wave mixing (FWM) in highly nonlinear fibers (HNLF) [1], Kerr effects in nonlinear interferometers [2], and cascaded second-order nonlinearities in periodically poled lithium niobate (PPLN) waveguides [3], where the input signal and output signal frequencies are identical. A new kind of PSA based on FWM in a HNLF has recently been experimentally demonstrated which provides phase-sensitive wavelength conversion (PS-WC) $[4,5]$. In that configuration, two idlers at two new wavelengths are generated in a phasesensitive fashion during a single stage of FWM interaction. Here, we report a novel scheme of PS-WC based on the combination of cascaded second-order nonlinear effects in two cascaded PPLN waveguides. PS-WC exploiting cascaded second-order nonlinearities in PPLN waveguides offers a number of advantages over the corresponding FWM-based approach. PPLN waveguides are compact and effectively immune to stimulated Brillouin scattering of the pump beams. Furthermore, contrary to fiber-based implementations, the operation of PS-WC exploiting cascaded second-order nonlinearities in PPLN waveguides is less susceptible to the generation of pump-pump and pump-signal harmonics [6], or to self-phase and cross-phase modulation [4].

\section{Operation principle}



Fig.1. Illustration of phase-sensitive wavelength conversion based on a combination of cSFG/DFG and cSHG/DFG in two cascaded PPLN waveguides.

Figure 1 illustrates the optical wavelength arrangement for our PS-WC scheme based on a combination of cascaded second-order nonlinearities in two PPLN waveguides. The quasi-phase-matching (QPM) wavelengths of the first and the second PPLN waveguide are detuned from each other, so as to place Pump 1 and the input signal symmetrically around the QPM wavelength of the first PPLN waveguide. Also the wavelength of Pump 1 coincides with the QPM wavelength of the second PPLN waveguide. The input signal, Pump 1 and Pump 2 are phase-locked relative to each other and launched into the first PPLN waveguide. In the cascaded sum frequency and difference 
frequency generation (cSFG/DFG) process occurring within the first PPLN waveguide, the input signal and Pump 1 generate the sum frequency (SF) by the SFG process, followed by the DFG process between the SF and Pump 2, generating the phase-preserving output at a new wavelength. Pump 2 is suppressed after the first PPLN waveguide, and the rest of the optical waves are subsequently launched into the second PPLN waveguide. In the cascaded second harmonic and difference frequency generation (cSHG/DFG) process occurring within the second PPLN waveguide, the second harmonic ( $\mathrm{SH}$ ) wave of Pump 1 is generated by the SHG process, and this is accompanied by the DFG process between the SH and the input signal which produces its phase-conjugate output. The outputs from cSHG/DFG and cSFG/DFG are inherently generated at the same wavelength, and after combination of these two outputs, the electric field at the output of the PPLN cascade can be approximated as

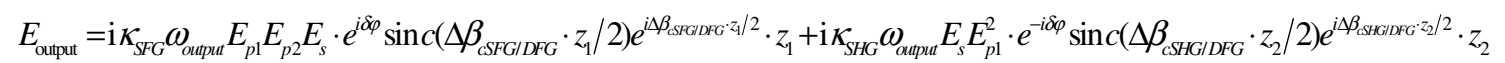

where $\kappa_{\mathrm{SHG}}$ and $\kappa_{\mathrm{SFG}}$ denote the nonlinear coupling coefficients of the SHG and the SFG process respectively, $\mathrm{E}_{\mathrm{p} 1}$, $\mathrm{E}_{\mathrm{p} 2}, \mathrm{E}_{\mathrm{s}}$ denote the electric field amplitudes of Pump 1, Pump 2 and the input signal respectively, $\delta \varphi$ denotes the relative phase between the input signal and the pumps, $z_{1}$ and $z_{2}$ are the lengths of the two PPLN waveguides respectively, and $\Delta \beta_{\mathrm{cSHG} / \mathrm{DFG}}$ and $\Delta \beta_{\mathrm{cSFG} / \mathrm{DFG}}$ are the phase mismatches of the cSHG/DFG process and the cSFG/DFG process respectively. If for simplicity we assume that the intensities of the two outputs are equalised, the phase mismatches of cSHG/DFG and cSFG/DFG are zero $\left(\Delta \beta_{\mathrm{cSHG} / \mathrm{DFG}}=\Delta \beta_{\mathrm{cSFG} / \mathrm{DFG}}=0\right)$, and the two waveguides have the same length $\left(z_{1}=z_{2}=z\right)$, then the electric field of the combined output can be described as

$$
E_{\text {output }}=\mathrm{i}_{S F G \text { output }} E_{p 1} E_{p 2} E_{s} z\left(e^{i}+e^{i}\right)
$$

Therefore, in the ideal case, the combination of the phase-conjugate output from cSHG/DFG and the phasepreserving output from cSFG/DFG results in perfect cancellation of the quadrature component of the output and amplification of the in-phase component as shown in Eq. 2.

\section{Experiments}

Fig. 2 shows the experimental setup used to realize our PS-WC scheme. Both of the PPLN waveguides (HC Photonics Corp.) were fiber-pigtailed, 30-mm long and with a SHG acceptance bandwidth of $0.4 \mathrm{~nm}$. The first PPLN waveguide operated at $50 \mathrm{C}$ with SHG QPM wavelength of $1546 \mathrm{~nm}$, while the second one operated at $60 \mathrm{C}$ with a SHG QPM wavelength of $1548 \mathrm{~nm}$. Two continuous wave $(\mathrm{CW})$ lasers were used as the signal source and Pump 1, operating at $1544 \mathrm{~nm}$ and $1548 \mathrm{~nm}$ respectively. The two beams were combined in a 3 -dB coupler, amplified and launched into $500 \mathrm{~m}$ of a HNLF to generate a phase-correlated idler wave at $1540 \mathrm{~nm}$ through FWM which was used as Pump 2. All of the optical waves were passed through Optical Processor 1 (OP1, Finisar Waveshaper 4000E), which was a programmable filter used to adjust the relative phase between the signal and the pumps and adjust the optical power of Pump 2, thereby controlling the strength of the cSFG/DFG process in PPLN1. After OP1, all of the optical waves were amplified and launched into the first PPLN waveguide which induced the cSFG/DFG process to generate the phase-preserving output at $1552 \mathrm{~nm}$. All of the resulting optical waves were subsequently passed through a second optical processor (OP2), which selectively suppressed Pump 2. After OP2, all of the optical waves were amplified and launched into the second PPLN waveguide which induced the cSHG/DFG process to generate the phase-conjugate output, also at $1552 \mathrm{~nm}$. Fig. 3(a) shows the measured spectra at the output of the second PPLN waveguide for the cases of maximum (red trace) and minimum conversion efficiency (blue trace) at the combined output. Note that these measurements were achieved by controlling the relative phase of the signal at $1544 \mathrm{~nm}$ in OP1. The wavelength conversion loss for the case of maximum conversion was $\sim 18 \mathrm{~dB}$. We define phase-sensitive swing (PSS) as the ratio between the maximum and minimum output power. The PSS of the combined output was $\sim 25.3 \mathrm{~dB}$. From Eq.1 we find that this corresponds to an amplitude ratio of the cSFG/DFG and cSFG/DFG outputs of $\sim 0.91: 1$. In contrast to the experimental results in [3], the large PSS of the combined output is due to the strong attenuation of the quadrature component of the signal rather than the amplification of the in-phase component. Fig. 3(b) shows the phase-sensitive behavior of the combined output plotted as a function of the relative phase between the input signal and the pumps. The experimental measurements (red spots) agree well with the

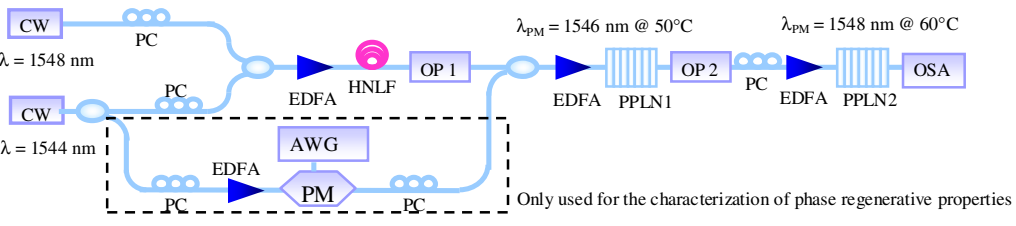

Fig.2. Experimental setup for the proposed PS-WC scheme based on a combination of cSHG/DFG and cSFG/DFG in two cascaded PPLN waveguides. CW: continuous wave, PC: polarization controller, EDFA: erbium-doped fibre amplifier, HNLF: highly nonlinear fibre, OP: optical processor, OSA: optical spectrum analyzer, PM: phase modulator, AWG: arbitrary waveform generator. 

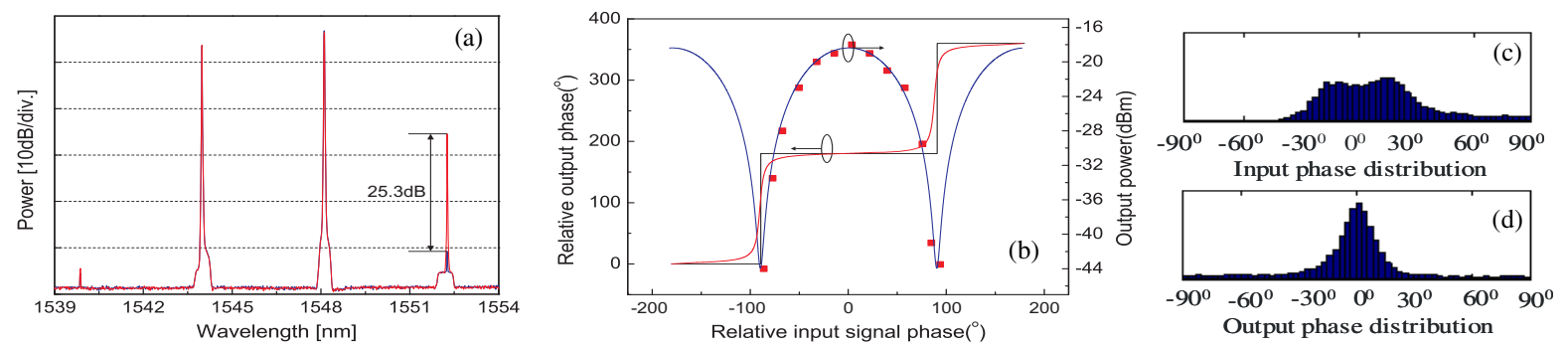

Fig.3. (a) Measured output spectra of the proposed PS-WC scheme showing the phase-sensitive swing. (b) The measured (red spots) and calculated phase-sensitive swing (blue curve) plotted as a function of the relative input signal phase, and the calculated input-output phase relationship of ideal case (black trace) and the proposed PS-WC scheme (red trace). (c) Measured phase distribution at the input and (d) the output.

theoretical curve (blue curve). The swing profile clearly indicates the phase-sensitive behavior with a period of $\pi$ phase. Fig. 3(b) also illustrates the calculated phase regenerative properties of our PS-WC scheme. The black trace shows the ideal case that the outputs from cSHG/DFG and cSFG/DFG are perfectly conjugated and of completely equal power. The perfect cancellation of the quadrature component of the signal allows close to ideal binary phase regeneration, independent of the overall cSHG/DFG and cSFG/DFG efficiency. The red trace shows the calculated phase regenerative properties of our PS-WC scheme for a PSS of 25.3dB as achieved in the experiment. Phase noise ranging between $\pm 30^{\circ}$ at an input bipolar (differential) phase-shift keying signal (D(B)PSK) can be suppressed to $\sim \pm 1.5^{\circ}$ at the output. As with all basic PSA configurations, phase regeneration in our PS-WC scheme is achieved at the expense of some added amplitude noise. In our PS-WC scheme, a phase fluctuation of $\pm 30^{\circ}$ is converted into an amplitude fluctuation of $0.62 \mathrm{~dB}$. (This amplitude noise can be reduced e.g. by increasing the conversion efficiency of the PPLN waveguides and saturating the gain of the outputs.) To characterize the phase regenerative capabilities of the system, the signal was split into two arms. The signal in the top arm was suppressed by OP1 after FWM, and a phase modulator was first used to emulate phase noise degradation of a binary phase modulated signal in the bottom arm. The modulator was driven by an electrical signal comprising the sum of binary (signal), and a broadband (noise) waveform, generated in a $24 \mathrm{GS} / \mathrm{s}$ arbitrary waveform generator. To measure the phase characteristics, the input signal and the idler were assessed using a homodyne coherent receiver. Because the homodyne receiver requires a local oscillator at the original signal wavelength, the output was first converted back to the signal wavelength using a parametric fiber wavelength converter with the output and Pump 1. Note that the use of homodyne detection allowed phase retrieval to be achieved without the need for a phase locked loop. The phase information was retrieved using digital signal processing, and the results are displayed in Fig. 3(c) and (d). We show phase histograms (20000 samples) of the input signal and the (converted) output. The input signal phase at the $0^{\circ}$ peak is distributed in a super Gaussian fashion with a $3 \mathrm{~dB}$ width of $\pm 30^{\circ}$, while at the output phase fluctuations have been considerably reduced, with a $3 \mathrm{~dB}$ distribution width of $<10^{\circ}$.

\section{Conclusion}

We have demonstrated a novel scheme of PS-WC based on a combination of the cSHG/DFG and cSFG/DFG processes in two cascaded PPLN waveguides. A PSS exceeding $25 \mathrm{~dB}$ was achieved in the experiment. Our first proof-of-principle experiment and the calculation of the phase regenerative properties also show the potential of our PS-WC scheme as a D(B)PSK phase regenerator.

\section{Acknowledgement}

The research leading to these results has received funding from the UK EPSRC under grant agreement EP/F032218/1.

\section{References}

[1] C. J. McKinstrie, and S. Radic, "Phase-sensitive amplification in a fiber," Opt. Express 12, 4973-4979 (2004).

[2] M. E. Marhic, C. H. Hsia, and J. M. Jeong, “Optical amplification in a nonlinear fiber interferometer,” Electron. Lett. 27, 210-211 (1991).

[3] K. J. Lee, F. Parmigiani, S. Liu, J. Kakande, P. Petropoulos, K. Gallo, and D. Richardson, "Phase sensitive amplification based on quadratic cascading in a periodically poled lithium niobate waveguide," Opt. Express 17, 20393-20400 (2009).

[4] K. Croussore, and G. Li, "Phase-regenerative DPSK wavelength conversion," in IEEE 20th annual meeting LEOS (Orlando, 2007).

[5] K. Croussore and G. Li, "Phase-regenerative wavelength conversion for BPSK and DPSK signals," IEEE Photon. Technol. Lett. 21, 70-72 (2009).

[6] C. J. McKinstrie, S. Radic, M. G. Raymer, and L. Schenato, "Unimpaired phase-sensitive amplification by vector four-wave mixing near the zero-dispersion frequency," Opt. Express 15, 2178-2189 (2007). 